\title{
Baryogenesis by R-parity violating top quark decays and neutron-antineutron oscillations
}

\author{
A.D. Dolgov ${ }^{a, b, c}$, F.R. Urban ${ }^{a, b, d}$ \\ a INFN, via Saragat 1, 44100, Ferrara, Italy \\ b University of Ferrara, Department of Physics, via Saragat 1, \\ 44100, Ferrara, Italy \\ c ITEP, Bol. Cheremushkinskaya 25, 117218, Moscow, Russia \\ d School of Physics and Astronomy, CAPT, University on Nottingham, \\ University Park, Nottingham NG7 2RD, United Kingdom
}

\begin{abstract}
Generation of the cosmological baryon asymmetry in SUSY based model with broken R-parity and low scale gravity is considered. The model allows for a long-life time or even stable proton and observable neutron-antineutron oscillations.
\end{abstract}

\section{Introduction}

Baryogenesis is well known to be a challenging issue in nowadays cosmology and particle physics. We know that, at least within a confidence radius of $10 \mathrm{Mpc}$, or even much more, see [1], the universe is strongly dominated by baryons, while practically no antibaryons are observed in the sky. The cosmological number density of baryons with respect to the number density of photons in cosmic microwave background radiation (CMBR) is quite small. From Big Bang Nucleosynthesis (BBN) and the angular fluctuations of CMBR, recently accurately measured by WMAP, we know that (see e.g. [2]):

$$
\beta=\frac{n_{b}-n_{\bar{b}}}{n_{\gamma}}=\frac{n_{B}}{n_{\gamma}} \approx 6 \cdot 10^{-10}
$$

On the other hand, this number is not so small, as it seems, compared to a locally baryo-symmetric universe, where the ratio $n_{b} / n_{\gamma}=n_{\bar{b}} / n_{\gamma}$ would have 
been some 10 orders of magnitude smaller than this. Explaining the origin of the cosmological asymmetry between particles and antiparticles and the value of ratio (11) has proven to be an interesting meeting point for particle physics and cosmology, especially since, according to the seminal paper by Sakharov [3], it requires and gives hints about physics beyond the standard model. Indeed, in order for most baryogenesis models to work, baryon number nonconservation, $\mathrm{C}$ and $\mathrm{CP}$ violation, and departure from thermal equilibrium are required, thus pushing forward the demand for new fundamental physics. In fact even within the context of the minimal standard model (MSM) these requirements are fulfilled, but the magnitude of the baryon asymmetry obtained in the frameworks of MSM is much smaller than the observed value (11).

There are many extensions of MSM which can explain the origin of the cosmological baryon asymmetry and its value, for a review see refs. [4, [5]. Doing that, one has to keep in mind a very strong lower bound on the proton life-time [2] $\tau_{p}>2 \cdot 10^{29}$ years (model independent) or $\tau_{p}>10^{31}-10^{33}$ years depending upon the decay channels. The goal of this work is to present a SUSY based model with broken $\mathcal{R}$-parity which allows for a long proton life-time, or even for stable proton and can explain the observed cosmological baryon asymmetry. As we see in what follows, it can be done in models with low scale gravity [6], where the short distance Planck mass $M^{*}$ is much smaller than the standard long distance Planck mass, $M_{P l}=1.2 \cdot 10^{19} \mathrm{GeV}$, due to propagation of gravity in higher dimensions ${ }^{1}$. There is also another possibility to have a low Planck mass in the early universe and the normal large one at later cosmological epochs, prior to primordial nucleosynthesis, due to time variation $M^{*}=M^{*}(t)$. The latter may be induced by the coupling $R f(\phi)$, where $R$ is the curvature scalar and $\phi$ is some time dependent scalar field [8]. As a by-product the suggested model may lead to potentially observable neutron-antineutron oscillations and B-nonconserving effects in processes with heavy quarks.

If indeed, the effective Planck mass in the early universe is about $\mathrm{TeV}$ values, we would be in trouble with many versions of baryogenesis scenarios which demand much higher energy for their realization, as e.g. GUT or baryo-through-lepto genesis. In this case SUSY theories with broken $\mathcal{R}$ parity present a viable possibility for successful cosmological baryogenesis and, what's more, they allow for some observable effects in particle physics with clear signatures and possibly non-negligible magnitudes.

In the next section the basic picture of the model is given, while the

\footnotetext{
${ }^{1}$ For a string theory inspired, altough non-supersymmetric, realisation of this model see ref. [7].
} 
third section is devoted to $\mathcal{R}$-parity violation. Afterward, in the fourth section, the decay channels of heavy particles which could potentially lead to cosmological baryon asymmetry are considered. In section five the out-ofequilibrium decays of massive particles in cosmological setup are discussed and the magnitude of baryon asymmetry is estimated. Furthermore, the processes in particle physics with the variation of the baryonic charge by two units, in particular neutron-antineutron oscillations and processes with heavy quarks are described (section 6). Finally, the conclusion is devoted to a brief summary of the results, and some ideas for future investigations.

\section{Basic features}

In this paper we investigate the possibility of generation of the observed baryon asymmetry at relatively low temperatures, around or below the Electroweak (EW) phase transition, in the context of the Minimal Supersymmetric Standard Model (MSSM), extended by an addition of $\mathcal{R}$-parity violating term in the superpotential, which simultaneously violates baryon-number conservation. In this section we give a qualitative picture of how such scenario could be realised, while in the subsequent sections we go through technicalities and details, in particular, we consider a concrete SUSY model with B-nonconservation.

Normally the models of low $T$ baryogenesis demand first order phase transition in the primeval plasma to break thermal equilibrium because the cosmological expansion rate, $H=\dot{a} / a$, at low $T$ is much smaller than the reactions rates. Indeed,

$$
H=\left(\frac{8 \pi^{3} g_{*}}{90}\right)^{1 / 2} \frac{T^{2}}{M_{P l}} \approx 16.6\left(\frac{g_{*}}{100}\right)^{1 / 2} \frac{T^{2}}{M_{P l}},
$$

where $g_{*}$ is the number of relativistic species contributing into the cosmological energy density; at $T \sim 100 \mathrm{GeV}$ it is about 100 in MSM and may be a factor 2 larger in MSSM. On the other hand, the characteristic rates of reactions between elementary particles are:

$$
\Gamma_{d} \sim \alpha N m, \text { and } \Gamma_{r} \sim 4 \pi \alpha^{2} N^{2} T
$$

where $\Gamma_{d}$ is the decay rate of a gauge boson with mass $m, \Gamma_{r}$ is the typical reaction rate, $\alpha \sim 0.01$ is the gauge coupling constant and $N$ is the number of open channels, it could be quite large, $N=10-100$.

Evidently, with $M_{P l} \sim 10^{19} \mathrm{GeV}$, deviations from equilibrium at electroweak scale for particles with $m \sim 100 \mathrm{GeV}$ would be about $10^{-17}$ which is 
surely not enough to create the baryonic excess (11). We see that in order for low temperature models to be efficient, it is necessary to require a lower Plank mass, which in turn may be provided by the same theories which present the necessary symmetries for keeping the proton stable enough, that is, extra dimensions and (super)string theories. Different models of baryogenesis with low scale gravity have been considered earlier in ref. 9].

Using eqs. (21) and (3) we find that thermal equilibrium would be strongly broken, i.e. $H / \Gamma \geq 1$, if the effective Planck mass is bounded from above by $M^{*}<10^{5} \mathrm{GeV}$. This estimate is obtained with $N \sim 10$ and $T \sim m \sim 100$ $\mathrm{GeV}$. If we demand a weaker condition, $H / \Gamma \geq 10^{-5}$, which may be still sufficient for generation of $\beta \sim 10^{-9}$, we would see that baryogenesis could be possible with $M^{*}<10^{10} \mathrm{GeV}$.

There could be a complication from another side: if the Hubble parameter is too large, then thermal equilibrium might never be created prior to baryogenesis. This may be even favorable for baryogenesis, as it was argued in the case of the normal $M_{P l}$ in ref. [10, where baryogenesis at reheating was considered. A slow process of preheating by the inflaton in the case of low effective Planck mass may be supplemented by gravitational particle production since the electroweak scale and the particle masses can now be close to $M^{*}$. Correspondingly heavy particles with $m \sim M^{*}$ could be efficiently created. Thus we can expect the primeval plasma enriched with heavy particles produced by gravity. An analysis of the gravitational particle production at the end of inflation can be found in refs. [11.

The initial properties of the cosmological plasma depend upon the magnitude of the Higgs field, $\phi$, during the process of (pre)heating. If $\phi$ had already reached its nonzero vacuum expectation value, then the produced particles would be massive. It is possible that the plasma temperature after thermalisation would be below the EW temperature, i.e. the plasma would be already in EW broken phase and the EW phase transition never occurred in cosmological history.

In what follows we neglect possible deviations off the standard thermal evolution and consider baryogenesis from initially equilibrium state. The nonequilibrium effects due to a possible overabundance of heavy particles, e.g. t-quarks, would lead to a larger baryon asymmetry.

Quite efficient breaking of thermal equilibrium in the scenario under consideration can be created by the following mechanism. At the temperatures above the EW phase transition all the Standard Model (SM) particles are massless (except for thermal masses, which are in any case lower than the temperature at this stage), as well as (possibly) light SUSY particles. At a certain stage the phase transition takes place, and the Higgs mechanism gives masses to SM particles via spontaneous symmetry breaking. Then, 
depending upon the final temperature after the transition, the now massive particles could get masses which would be higher than the temperature after the phase transition, while some other would remain effectively massless with $m<T$. The crucial temperature is known by the standard calculations to be related to the Higgs mass, (see e.g. book [12]). Given the present day lower limit on the Higgs mass, we see that the only SM particle which may have a mass above the final temperature after the EW phase transition is the top quark. We believe that SUSY partners acquire their masses through soft symmetry breaking by the analogous Higgs effect at the same EW energy scale.

If the phase transition is fast enough, so that the $t$-quark would not decay down to the Boltzmann suppressed equilibrium density at $m_{t}>T$ in the course of the phase transition, then the system would arrive to the state when the number density of $t$-quarks at the end of the EW phase transition would be equal to the number density of massless particles and grossly exceed their equilibrium value. This makes favorable conditions for baryogenesis through $t$-quark decays which ultimately would reduce their amount down to the equilibrium value.

The rate of the phase transition is determined by the evolution of the temperature dependent Higgs mass:

$$
m_{\phi}^{2}(T)=g_{t}^{2} T^{2} / 2-m_{0}^{2} / 2
$$

where $m_{0}$ is the vacuum Higgs mass and $g_{t} \sim 1$ is the Yukawa coupling of Higgs boson to $t$-quark. In the course of the phase transition the temperature drops down roughly speaking by $\Delta T \sim m_{0}$. According to the standard law of cosmological temperature red-shift, $\dot{T}=-H T$, we find that the time of the p.t. is about $\delta t \sim H^{-1}(\Delta T / T) \sim H^{-1}$. In realistic case the law of temperature variation is different, due to presence of the time varying Higgs condensate, but not much. On the other hand, the decay width of $t$-quark is about $\Gamma_{t} \sim \alpha m_{t}$, (see eq. (19)). Thus the decay rate would be slow in comparison with the rate of the p.t. if

$$
M^{*}<10^{3} m_{t} \approx 10^{5} \mathrm{GeV}
$$

For a bigger $M^{*}$ the $t$-quark decay would be faster than the rate of the p.t. and its number density would follow the equilibrium law, $n_{t} \sim \exp \left(-m_{t} / T\right)$. The baryogenesis through $t$-quark decays would not be efficient if $T / m_{t} \ll 1$, but if this ratio is only mildly smaller than unity then $B$-nonconserving decays of $t$-quark might be efficient enough to create the observed $\beta$. Moreover, $t$-quarks could decay in the course of the p.t. creating baryon asymmetry on the way. 
Thus we see that if some of $t$-quark decay channels, or decay channels of the produced particles, can accommodate baryon number violation as well as CP nonconservation, we have fulfilled all the three basic Sakharov's requirements for a successful baryogenesis. In our model, allowing for B-violation coupling in the superpotential, we provide the possibility for the asymmetry to be generated, if at least one superquark is lighter than the top quark, so that some B-violating channels are kinematically allowed. It is also possible to account for a baryon asymmetry in the situation in which the abovementioned superquarks are (slightly) heavier than the top quark, but in this case a more detailed analysis of the mechanism through which the superpartners acquire their masses is needed. Let us remind that baryogenesis in the minimal standard model happens to be unsuccessful, in particular, because of very weak CP-violation in the MSM, see e.g. the lectures [13]. In a SUSY extended model CP-violation is not restricted by the CKM matrix and can be much stronger. We neglect electroweak nonconservation of baryons induced by sphalerons because by construction baryogenesis proceeded below the EW p.t.

In what follows we retread in detail every step outlined above. We first consider the presence of the baryon-number-violating term in the superpotential from a theoretical point of view, and discuss some of its phenomenological consequences. Then we will write down the trees of the essential processes, and estimate the baryon asymmetry in each case, in a purely particle physics way (no plasma, no expansion). Afterward, we will set these estimates in a real cosmological environment showing how the necessity of a lower Plank mass arises, and find that the asymmetry previously estimated is indeed close to the actual one. Finally, keeping in mind restrictions on the model parameters necessary for successful generation of the baryon asymmetry, we will discuss the predictions of the model for B-nonconserving processes in particle physics, i.e. neutron-antineutron oscillations and heavy quark decays, and conclude.

\section{SUSY background}

The $\mathcal{R}$-parity violating superpotential, consistent with the gauge symmetries of the SM, as well as with global supersymmetry, made up only of MSSM fields, can be written as

$$
W_{\mathcal{R}}=\mu^{i} L_{i} H_{u}+\frac{1}{2} \lambda^{i j k} L_{i} L_{j} E_{k}^{c}+\lambda^{\prime i j k} L_{i} Q_{j} D_{k}^{c}+\frac{1}{2} \lambda^{\prime \prime i j k} U_{i}^{c} D_{j}^{c} D_{k}^{c},
$$

where $H_{u}, L, E^{c}, Q, U^{c}$, and $D^{c}$ are the up-type Higgs, left- and right-handed lepton, left- and (up- and down-) right-handed quark chiral supermultiplets; 
the superscript $c$ denotes charge conjugation, and we have suppressed all fermion, colour and gauge indices, see e.g. [14, 15]. We notice at a glance that these couplings violate either baryon or lepton number separately, in particular, the first three terms proportional to $\mu, \lambda$ and $\lambda^{\prime}$ have $\Delta L=1$, while the fourth with coupling $\lambda^{\prime \prime}$ has $\Delta B=-1$, and is the one which we will later focus our attention on. For applications of lepton number violating terms within the context of leptogenesis see ref. [16]. The $\lambda^{\prime \prime}$ term in the superpotential (6) leads to the following explicit interaction Lagrangian ${ }^{2}$ :

$$
\mathcal{L}_{i n t}=-\frac{1}{2} \lambda^{\prime \prime i j k}\left(\tilde{u}_{i}^{*} \bar{d}_{j} d_{k}^{c}+\tilde{d}_{k}^{*} \bar{u}_{i} d_{j}^{c}+\tilde{d}_{j}^{*} \bar{u}_{i} d_{k}^{c}\right)+\text { h.c. }
$$

where the $*$ denotes the complex conjugation of a scalar field, and h.c. means hermitian conjugate, and where again fermion, colour and gauge indices are suppressed. It is essential for the future estimates that $\lambda_{i j k}^{\prime \prime}$ is antisymmetric with respect to the last two indices $j$ and $k$, because of the antisymmetry with respect to colour.

$\mathcal{R}$-parity is known to be defined as:

$$
\mathcal{R}=(-1)^{R}=(-1)^{3(B-L)+2 s}
$$

where $B, L$ and $s$ are baryon number, lepton number, and spin, respectively. It is straightforward to see that if $\mathcal{R}$-parity is exactly conserved, then every term in $W_{\mathcal{R}}$, eq. (6) , is strictly forbidden. However, it is not necessary from a phenomenological point of view to forbid simultaneously all the couplings in (6), since, for instance, the disastrously efficient tree-level diagrams for proton decay are not generated if only the $\lambda^{\prime \prime}$ term is present. Moreover in such a theory proton must be absolutely stable. Indeed in this case the lepton number is strictly conserved and proton simply does not have any channel to decay into ${ }^{3}$. On the other hand, neutron-antineutron oscillations and other processes with variation of baryonic number by two, e.g. nuclei decays, may become observable with a mild increase of the present-day experimental accuracy. If in addition to $\lambda^{\prime \prime}$ other couplings in eqs. (6)77) are also nonvanishing they would induce jointly proton decay but one can always take them small enough to avoid conflicts with experimental bounds.

The discussion of all the different combinations of vertices, with their experimental consequences as well as the parities associated to them and the

\footnotetext{
${ }^{2}$ Note that this is not complete since we must include soft SUSY breaking terms consistent with the above mentioned symmetries, see ref. [15.

${ }^{3}$ In higher orders of perturbation theory proton may decay due to a possible small Majorana mass of neutrino or due to exchange of the heavy Majorana fermion responsible for see-saw mechanism, if they exist.
} 
underlying theories which provide these symmetries, is beyond the scope of this paper, and we address the interested reader to the comprehensive review [15], in particular to section 2.7, references therein, and ref. [17. The main point which we mention here is that we can build a phenomenologically acceptable SUSY model with a superpotential which includes the $\lambda^{\prime \prime}$ term, and forbids all the $\Delta L \neq 0$ ones. These models are usually embedded in (super)string theories, in which the way the extra dimensions are compactified, or, in the case of braneworld, the internal symmetries between the branes, provide the necessary symmetries for this configuration to be realised.

The next point which deserves discussion is the issue of the mass spectrum of the MSSM. Since none of the superpartners of the known SM particles are observed experimentally, we have only lower limits on their masses which can be found in ref. 2]. Theoretically there is no certainty about the form of the spectrum, but it is widely believed that one of the two mass-eigenstates of s-tops and/or one out of the two of s-bottoms are lighter than all the other s-quarks, and that some neutralinos and/or charginos are likely to be even lighter.

Lacking precise knowledge in this sector, we assume as an exemplificative toy model a given spectrum compatible with experimental data, and with some general requirements on SUSY models. To be more precise, we assume either that there is one out of the two mixtures of s-tops, or one of the two mixtures of the s-bottoms, which is lighter than the ordinary top quark, while all others s-quarks are heavier. We will specify later, when we consider quantitatively some concrete model, where the neutralinos and charginos reside in this scenario, since different possibilities are allowed and lead to (slightly) different results.

As a final remark let us note that if we take the lower limits, given by the Particle Data Group [2], for the superpartner masses ${ }^{4}$, whatever couple of (different) particles we choose, their total mass, $m_{1}+m_{2}$, is either very close to or mostly above, the top quark mass. Actually, the only combinations with $m_{1}+m_{2}<m_{t}$ we can find are $\tilde{\chi}_{1}^{0}$, or $\tilde{\chi}_{2}^{0}$, and one light s-quark or chargino $^{5}$. Thus we should not consider decay channels of top quark and daughter particles where more than one s-particle are produced, with the exceptions mentioned above, since it appears unlikely that these channel are kinematically allowed.

\footnotetext{
${ }^{4}$ Actually these limits have been found assuming $\mathcal{R}$-parity conservation, but we believe that possible $\mathcal{R}$-parity non-conserving decays do not change these bounds significantly.

${ }^{5}$ Throughout the paper we denote super-quarks as s-quarks, which should not be confused with the strange MSM quark.
} 


\section{Particle physics models and possible decay channels}

We outline here four different possibilities of heavy particle decay channels determined by a different choice of their mass relations. Below we draw a schematic tree for the processes in every situation, and compute the baryonantibaryon asymmetry generated by these decays assuming freely decaying particles, thus neglecting the universe expansion and the thermal bath in which particles are immersed, where, given the latter, we are neglecting inverse reaction too. At this stage we assume, moreover, that SUSY particles are produced only by decays of heavy SM particles and their daughter processes. In the realistic cosmological situation when the $t$-quark became out of equilibrium after the EW p.t. because of $m_{t}>T$, as is described in sec. 2, the inverse decays induced by the superpartners already present in the plasma would not be essential because of the Boltzmann barrier and thus the assumption of absence of s-particles in the initial state is justified.

To be more specific, after the EW p.t. there would be heavy t-quarks with $m_{t}>T$ but with a non-suppressed number density. There could be some other massive SUSY partners as well, also with $m_{s}>T$. If $m_{s}>m_{t}$, then their decays could contribute to the baryon asymmetry. There are also some lighter particles, into which t-quark may decay. The inverse decay and resonance reactions with these particles may diminish the asymmetry. However, since light particles thermalise quickly, their number density would be close to that of massless particles, i.e. $n \sim T^{3}$, and their spectrum would be the usual equilibrium one with this $T$. If this is the case, though it is not necessary so, then the inverse decay and resonance scattering of light particles would not be important, because the probabilities of these processes are small, $\sim \exp \left(-m_{t} / T\right)$, while the number density of t-quarks is much larger than $\left(m_{t} T\right)^{3 / 2} \exp \left(-m_{t} / T\right)$ Hence, for every decay process, we neglect additional (inverse and resonance scattering) reactions while considering the cosmological setup. A more accurate study of the complete kinetics of B-nonconservation in the primeval plasma may possibly change the simple estimates presented below but not by a large factor.

In the next section we estimate the baryon asymmetry generated by tquark decays and show that the results are essentially the same as presented in subsections 4.1 and 4.2 . once we set the Plank mass in a certain low-scale range. 


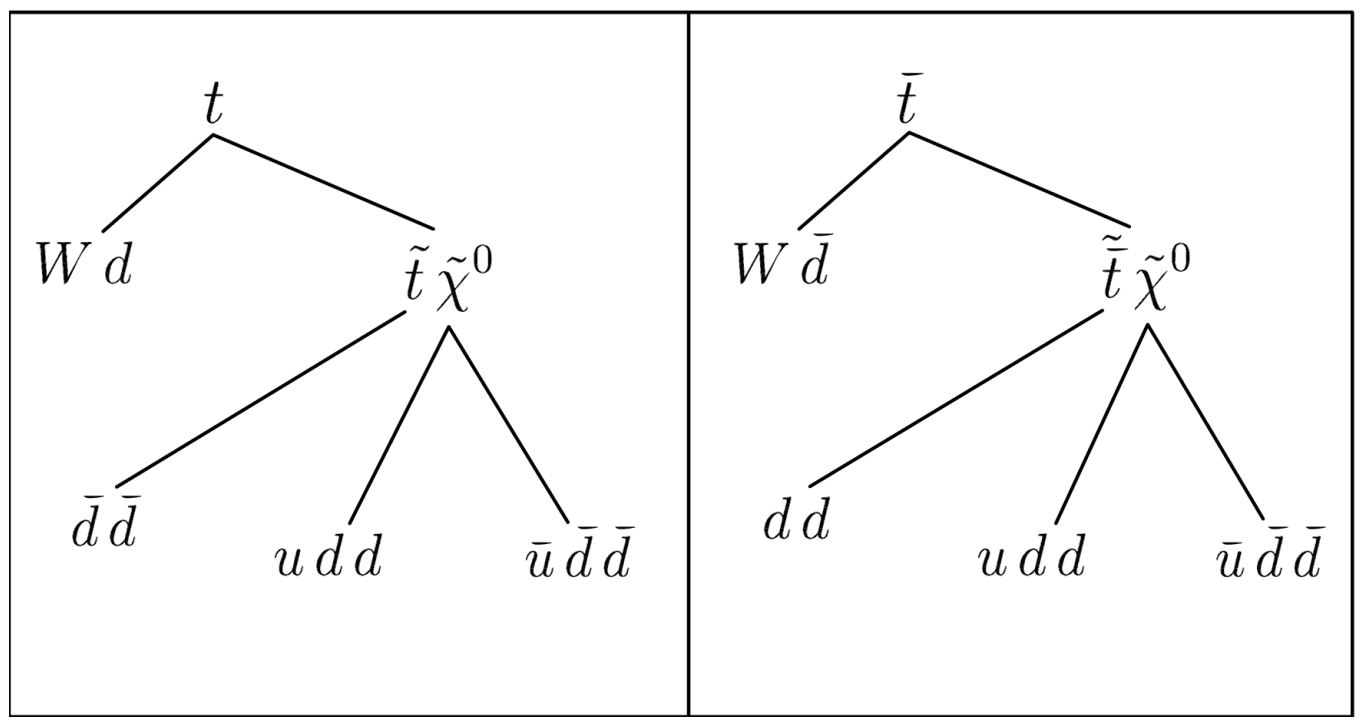

Figure 1: Schematic representation of the processes involved. As stated in footnote[ ' $\mathrm{u}$ ' and 'd' stand for generic up- and down-type quarks, while 't' and 'b' are the third family top and bottom quarks. Note however that not every combination of quarks is allowed, see the discussion in section [5

\subsection{Lightest s-top}

Let us first consider the scenario in which the only SUSY particles lighter than the top quark are the s-top, $\tilde{t}_{1}$, and the lightest neutralino, $\tilde{\chi}_{1}^{0}$, where the subscript means that we are dealing with only one mixture (labeled 1) of a certain kind of s-particles, which is supposed to be the lightest. We begin with an equal initial number of top quarks and antiquarks alone, and study the evolution of this system. If $m_{\tilde{t}_{1}}+m_{\tilde{\chi}_{1}^{0}}<m_{t}$, then the possible processes' tree is shown in fig. 1. 
We parametrise the branching ratios in the following way ${ }^{6}$ :

$$
\begin{aligned}
\Gamma\left(t \rightarrow W^{+}+d\right) / \Gamma_{t}^{t o t} & =1-x \\
\Gamma\left(\bar{t} \rightarrow W^{-}+\bar{d}\right) / \Gamma_{\bar{t}}^{t o t} & =1-\bar{x} \\
\Gamma\left(t \rightarrow \tilde{t}_{1}+\tilde{\chi}_{1}^{0}\right) / \Gamma_{t}^{t o t} & =x \\
\Gamma\left(\bar{t} \rightarrow \tilde{\bar{t}}_{1}+\tilde{\chi}_{1}^{0}\right) / \Gamma_{\bar{t}}^{t o t} & =\bar{x} \\
\Gamma\left(\tilde{t}_{1} \rightarrow d+d\right) / \Gamma_{\tilde{t}}^{t o t} & =1 \\
\Gamma\left(\tilde{\bar{t}}_{1} \rightarrow \bar{d}+\bar{d}\right) / \Gamma_{\tilde{t}}^{t o t} & =1 \\
\Gamma\left(\tilde{\chi}_{1}^{0} \rightarrow u+d+d\right) / \Gamma_{\tilde{\chi}}^{t o t} & =\frac{1-\epsilon_{\tilde{\chi}}}{2} \\
\Gamma\left(\tilde{\chi}_{1}^{0} \rightarrow \bar{u}+\bar{d}+\bar{d}\right) / \Gamma_{\tilde{\chi}}^{t o t} & =\frac{1+\epsilon_{\tilde{\chi}}}{2}
\end{aligned}
$$

The last four processes do not conserve baryonic charge and, in the absence of $\mathcal{R}$-parity breaking interaction (6), $\tilde{t}_{1}$ and $\tilde{\chi}_{1}$ would be stable. The difference between $x$ and $\bar{x}$ is induced by CP-violation and is small because CP-breaking can manifest itself only in higher orders of perturbation theory due to rescattering in the final state, see e.g. [13, 21]. The same result applies to $\epsilon_{\tilde{\chi}}$, which is non-zero but small.

Now, if we assume that these particles decay freely and inverse processes are negligible, we find that the final asymmetry generated after several characteristic lifetimes of the decaying particles, is given by summing all the quarks and antiquarks in the final states, with their respective baryonic charges, multiplied by the branching ratio of the channel into which they have been produced. This gives:

$$
\Delta B=(\bar{x}-x)-\epsilon_{\tilde{\chi}}(\bar{x}+x) \simeq \epsilon_{t}
$$

where $\epsilon_{t}=\bar{x}-x$, and in the last equality we have retained only the first order terms, since we expect $\bar{x}, x$ as well as $\epsilon_{\tilde{\chi}}$ to be small.

If no CP violation is allowed in the standard model particle decay, $t \rightarrow$ $W+q$, which means that $\bar{x}=x$, we obtain:

$$
\Delta B=-2 x \epsilon_{\tilde{\chi}}
$$

\footnotetext{
${ }^{6}$ Here and in what follows ' $u$ ' and 'd', together with their antiparticles and superpartners, mean up- and down-type quarks of arbitrary family, respectively, while ' $t$ ' and 'b' stand for top and bottom quarks of the third family; again the definition extends to antiparticles and superpartners.
} 


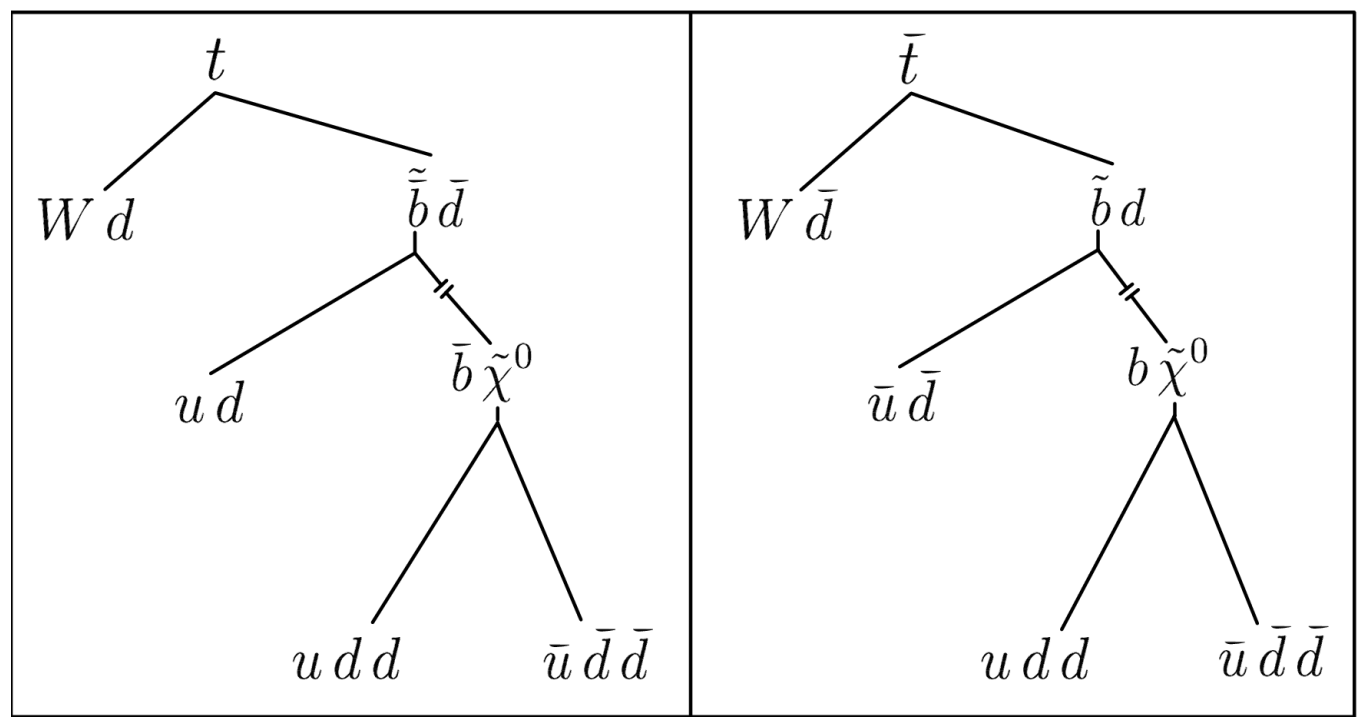

Figure 2: Schematic representation of the processes involved. As stated in footnote [6] ' $u$ ' and 'd' stand for generic up- and down-type quarks, while 't' and 'b' are the third family top and bottom quarks. Note however that not every combination of quarks is allowed, see the discussion in section $\left[\right.$ The line going from $\tilde{b}$ to $b \tilde{\chi_{0}}$ is crossed to indicate that this channel may be closed if the mass difference does not allow it.

that is, the asymmetry is entirely generated in the second step $^{7}$, of the CPviolating decays of neutralinos.

Concluding this subsection we notice that if the condition $m_{\tilde{t}_{1}}+m_{\tilde{\chi}_{1}^{0}}<m_{t}$ is no longer satisfied because of a heavier either neutralino or s-top, then the baryon asymmetry cannot be generated in this version of the scenario.

\subsection{Lightest s-bottom}

We turn now to the case where the lightest s-quark is the s-bottom $\tilde{b}_{1}$, and the mass spectrum goes as:

$$
m_{\tilde{\chi}_{1}^{0}}<m_{\tilde{b}_{1}}<m_{t}<m_{\tilde{t}_{1}}
$$

Let us assume again that there are some equal initial numbers of top and antitop quarks and no other particles are present. In this case the baryon asymmetry can be generated in three steps by the decay of t-quarks and their decay products', as sketched in fig. 2. The last step is actually realised only if the chain of inequalities (11) is true all the way down. In fact, if the neutralino

\footnotetext{
${ }^{7}$ It is easy to see that even if we have more than one channel with $\Delta B \neq 0$, as in fact we have, no asymmetry develops in the primary decay of the top quark, since all these B-violating channels have the same $\Delta B$.
} 
is heavier than the $\tilde{b}_{1}$, the tree of decays changes, because the decay channel $\tilde{\bar{b}}_{1} \rightarrow \bar{b}+\tilde{\chi}_{1}^{0}$ and its charge conjugated one are no longer kinematically allowed, and then this process, as well as the subsequent neutralino decay, must be cut from the tree, leaving only the $\Delta B \neq 0$ channel.

Then, as in the previous case, the branching ratios can be written as follows:

$$
\begin{aligned}
\Gamma\left(t \rightarrow W^{+}+d\right) / \Gamma_{t}^{t o t} & =1-x \\
\Gamma\left(\bar{t} \rightarrow W^{-}+\bar{d}\right) / \Gamma_{\bar{t}}^{t o t} & =1-\bar{x} \\
\Gamma\left(t \rightarrow \tilde{\bar{b}}_{1}+\bar{d}\right) / \Gamma_{t}^{t o t} & =x \\
\Gamma\left(\bar{t} \rightarrow \tilde{b}_{1}+d\right) / \Gamma_{\bar{t}}^{t o t} & =\bar{x} \\
\Gamma\left(\tilde{\bar{b}}_{1} \rightarrow \bar{b}+\tilde{\chi}_{1}^{0}\right) / \Gamma_{\overline{\bar{b}}}^{t o t} & =1-y \\
\Gamma\left(\tilde{b}_{1} \rightarrow b+\tilde{\chi}_{1}^{0}\right) / \Gamma_{\tilde{b}}^{t o t} & =1-\bar{y} \\
\Gamma\left(\tilde{\bar{b}}_{1} \rightarrow u+d\right) / \Gamma_{\bar{b}}^{t o t} & =y \\
\Gamma\left(\tilde{b}_{1} \rightarrow \bar{u}+\bar{d}\right) / \Gamma_{\tilde{b}}^{t o t} & =\bar{y} \\
\Gamma\left(\tilde{\chi}_{1}^{0} \rightarrow u+d+d\right) / \Gamma_{\tilde{\chi}}^{t o t} & =\frac{1-\epsilon_{\tilde{\chi}}}{2} \\
\Gamma\left(\tilde{\chi}_{1}^{0} \rightarrow \bar{u}+\bar{d}+\bar{d}\right) / \Gamma_{\tilde{\chi}}^{t o t} & =\frac{1+\epsilon_{\tilde{\chi}}}{2}
\end{aligned}
$$

Here again the last four processes violate baryonic number conservation but in addition the processes 3 and 4 also break it. We see that, with respect to the previously considered scenario, here all the three steps can generate an asymmetry, since all of them include two different decay channels with different $\Delta B$, either $\Delta B_{c h .1}=0$ and $\Delta B_{c h .2}= \pm 1$ (first and second steps), or $\Delta B_{c h .1}=1$ and $\Delta B_{c h .2}=-1$ (third step).

We have chosen this parametrisation since we expect the ratio between $\tilde{b}_{1} \rightarrow \bar{u}+\bar{d}$ and $\tilde{b}_{1} \rightarrow b+\tilde{\chi}_{1}^{0}$ to be small ${ }^{8}$, which in turn means that $\bar{y}$ and $y$ are small, and this gives us a convenient way to express the baryon asymmetry generated as:

$$
\Delta B=(\bar{x}-x)-(\bar{x} \bar{y}-x y)-\epsilon_{\tilde{\chi}}(\bar{x}+x-(\bar{x} \bar{y}+x y)) \simeq \epsilon_{t},
$$

where again we could focus on the case $\bar{x}=x$ to obtain:

$$
\Delta B=-x\left((\bar{y}-y)+\epsilon_{\tilde{\chi}}(\bar{y}+y-2)\right) \simeq-x\left(\epsilon_{\tilde{b}}-2 \epsilon_{\tilde{\chi}}\right)
$$

\footnotetext{
${ }^{8}$ The estimates of the decay rates and the discussion on that will be given in the next section.
} 
with $\epsilon_{\tilde{b}}$ being $\bar{y}-y$, and, as in the previous subsection, the last equalities in (12) and (13) follow, once one discards higher-order terms, given the smallness of $\bar{x}, x, \bar{y}, y$ and $\epsilon_{\tilde{\chi}}$.

Actually, as we see in what follows, it is not mandatory that $\bar{y}$ and $y$ are small. Indeed, if this is not the case, we might want to change slightly our parametrisation of the branching ratios, and redefine $\bar{y}$ and $y$ as $1-\bar{z}, 1-z$, respectively, where now $\bar{z}$ and $z$ are small, and this gives the following result:

$$
\Delta B=(\bar{x} \bar{z}-x z)-\epsilon_{\tilde{\chi}}(\bar{x} \bar{z}+x z)
$$

or, with no $\mathrm{CP}$ violation in the SM decays, given $\bar{z}-z=\epsilon_{\tilde{b}^{\prime}}=-\epsilon_{\tilde{b}}$ :

$$
\Delta B=x(\bar{z}-z)-x \epsilon_{\tilde{\chi}}(\bar{z}+z) \simeq x \epsilon_{\tilde{b}^{\prime}}
$$

Note that if instead the case of a heavy neutralino is considered, we must set $y=\bar{y}=1$, or equivalently $z=\bar{z}=0$. Doing so we find that a net asymmetry cannot develop, thus if these scenarios are to work we must demand (at least) one light neutralino. As a final comment concluding this subsection we point out that there are no kinematically allowed processes involving charginos.

\section{Cosmological setting}

In this section we analyse the evolution of an initial equal number density of top and antitop quarks in cosmological plasma. We begin by discussing initial conditions, then we estimate the decay rates for every process, and study the cosmological evolution of the baryon asymmetry. Since the two possibilities coming from different mass spectra described above have similar outcome, we will focus only on the second scenario, namely $m_{\tilde{\chi}_{1}^{0}}<m_{\tilde{b}_{1}}<m_{t}<m_{\tilde{t}_{1}}$.

As described in section 2, before the EW phase transition all SM particles were massless, except for small thermal masses, since the symmetry was unbroken. Following the standard calculations, as given for instance in ref. 12, we find the relation which links the characteristic temperature after the transition with the Higgs mass

$$
T_{p . t .}^{2} \simeq 1.5\left(m_{H}^{2}+(44 \mathrm{GeV})^{2}\right)
$$

This relation in fact should be corrected in the context of MSSM, because possibly also SUSY particles would take part in the EW transition, but the effects are not very strong [18].

Since we require the temperature after the transition to be lower than the top quark mass, $m_{t} \approx 175 \mathrm{GeV}$, we have the upper bound on the Higgs 
boson mass $m_{H} \leq 135 \mathrm{GeV}$, while with the current experimental lower bound $m_{H}>114.4 \mathrm{GeV}$, we find a temperature around $150 \mathrm{GeV}$. Within this range of Higgs masses, after the EW phase transition the top quarks and antiquarks are the only SM particles which are truly non-relativistic (or better to say, semi-relativistic), thus they are interesting candidates for the "parents" of out-of-equilibrium decay. However we could relax this hypothesis allowing for a wider range of Higgs masses, but in that case deviations from equilibrium are smaller. Nevertheless a reasonable net asymmetry will develop even in this case.

We proceed through the detailed calculations of the requirements for this model to work, completing the discussion given in section 2. In order to know if the reactions which we are interested in, are in thermal equilibrium or not, we must estimate their decay rates and compare them with the expansion rate of the universe, given by the Hubble parameter $H=\dot{a} / a$, when $T \approx m$, where $m$ is the mass of the decaying particle. If $\Gamma / H \gg 1$, then equilibrium would be established, while if

$$
\Gamma / H \ll 1
$$

the decay would be "frozen".

The second point we must look at is the stability of top quarks and antiquarks, since we don't want these particles to decay before the EW transition. This is because otherwise, once the transition takes place, all the $t$ and $\bar{t}$, now massive, would decay almost instantaneously, and, since they are non-relativistic, their number density would be exponentially suppressed. Hence, no asymmetry will arise. This requirement can be written as

$$
\Gamma_{\text {tot }} t_{\text {p.t. }} \ll 1
$$

that is, the characteristic time of the EW phase transition must be much less than the mean life of top and antitop quarks.

We note here that the above stated requirements of out-of-equilibrium decay and long lived particle, in standard cosmology result almost the in same numerical constraints, since $H=1 /(2 t)$ or $H=2 /(3 t)$ in radiation or dust dominated universes, respectively. Then, when we fix the temperature $T$ we have also fixed the Hubble parameter $H$ and the time $t$ at the, almost, same numerical value. However this is no longer true if we consider e.g. brane worlds or loop quantum gravity cosmologies, and, without going that far, even an universe dominated by an inflaton in its oscillating regime. In non standard cosmologies $H^{2} \neq \rho$ with $\rho$ being the energy density of the dominant component which fills the universe, generally $H^{2} \sim L(\rho)$ and therefore $H \neq$ $t^{-1}$, see reviews [19, 20] and references therein. In the case of an oscillating 
inflaton dominated universe, we have instead that $H \sim T^{4}$, which again gives a different relation between time and temperature, which however depends on the specific inflationary model, see 22 . In both these two cases we have two different numerical constraints for our requirements (17) and (18), but in what follows we assume the normal evolution specified at the beginning of this paragraph.

At this point we have formulated the two basic requirements on the decay rates, then we list below the rates we will use afterwards.

$$
\begin{aligned}
\Gamma(t \rightarrow W+d) & =\frac{g^{2}}{64 \pi} \frac{V_{t d}^{2}}{m_{W}^{2}} m_{t}^{3} \\
\Gamma\left(t \rightarrow \tilde{\bar{b}}_{1}+\bar{d}\right) & =\frac{\lambda_{t b d}^{\prime \prime 2}}{128 \pi} Z_{\tilde{b}_{1}}^{2} m_{t} \\
\Gamma\left(\tilde{\bar{b}}_{1} \rightarrow \bar{b}+\tilde{\chi}_{1}^{0}\right) & \approx \frac{g^{2}}{10^{2} \pi} Z_{\tilde{b}_{1}}^{2} Z_{\tilde{\chi}_{1}^{0}}^{2} m_{\tilde{b}_{1}} \\
\Gamma\left(\tilde{\bar{b}}_{1} \rightarrow u+d\right) & =\frac{\lambda_{u b d}^{\prime \prime 2}}{64 \pi} Z_{\tilde{b}_{1}}^{2} m_{\tilde{b}_{1}} \\
\Gamma\left(\tilde{\chi}_{1}^{0} \rightarrow u+d+d\right) & \approx \frac{g^{2}}{10^{4} \pi^{3}} \frac{\lambda_{u d d}^{\prime \prime 2} Z_{\tilde{\chi}_{1}^{0}}^{2}}{m_{\tilde{q}}^{4}} m_{\tilde{\chi}_{1}^{0}}^{5}
\end{aligned}
$$

where: $g$ is the EW coupling constant, $Z_{i}$ is a function of the $\mathrm{i}$-th SUSY particle mixing parameters, $m_{\tilde{q}}$ is the mass scale of the heavy superquarks, and $\lambda_{i j k}^{\prime \prime}$ is the $\mathcal{R}$-parity violating coupling constant relative to the B-violating part of the superpotential (6). As before, "u" and "d", mean up- and downtype quarks, respectively ${ }^{9}$, while $\mathrm{t}$ and b stand for top and bottom quarks; the definition extends to antiparticles and their (anti)superpartners.

Let us now turn to consideration of the demands we want to satisfy. We will use the following numerical values. The top quark mass is $m_{t}=175 \mathrm{GeV}$, the $\mathrm{W}$ mass is $m_{W}=80.4 \mathrm{GeV}$, the heavy s-quarks mass scale is $m_{\tilde{q}} \approx 300$ $\mathrm{GeV}, Z_{\tilde{b}_{1}}^{2}=Z_{\tilde{\chi}_{1}^{0}}^{2}=0.5$, though those values are unknown and could well be one order of magnitude lower. We set the masses of the unknown light s-particles to the values $m_{\tilde{b}_{1}} \approx 100 \mathrm{GeV}$ and $m_{\tilde{\chi}_{1}^{0}} \approx 50 \mathrm{GeV}$. Furthermore, from now on we will drop the apices on $\lambda^{\prime \prime}$, bearing in mind that it refers to the baryon number-violating term in the superpotential.

Up to this point we have not specified the range which the B-violating $\lambda$ couplings could span. We have different processes in which almost every different possible $\lambda$ coupling is involved. More specifically, in process (20) we

\footnotetext{
${ }^{9}$ Not every combination is allowed, however. For instance in the process $t \rightarrow \tilde{\bar{b}}_{1}+\bar{d}$, only $\mathrm{d}=$ down and $\mathrm{d}=$ strange are allowed by the symmetries of the coupling matrices, once one takes into account colour.
} 
can have $\lambda_{331}$ or $\lambda_{332}$, the sub-numbers being the family which the quarks belong to, where the first number refers to the up-type quark involved, and the other two refer to the two down-type quarks, with $\lambda_{i j k}=-\lambda_{i k j}$. Then, in process (22) the possibilities are $\lambda_{113}, \lambda_{123}, \lambda_{213}$ or $\lambda_{223}$, while neutralino decay, (23), splits into six different processes determined by $\lambda_{112}, \lambda_{113}, \lambda_{123}$, $\lambda_{212}, \lambda_{213}$ or $\lambda_{223}$. Referring again to the review [15] (sec. 6), we see that some of these couplings, are quite strongly constrained, e.g. $\lambda_{112}<10^{-6}$, $\lambda_{113}<10^{-3}$, while the other could be well above unity. The most stringent bounds on $\lambda_{i j k}$ are obtained from nucleus stability which is destroyed by the baryon-antibaryon transformation. It is discussed in the next section in connection with neutron-antineutron oscillations.

The huge dispersion in the values of the $\mathcal{R}$-parity -breaking couplings $\lambda_{i j k}$ may be related to the similar or larger dispersion in the Yukawa couplings of Higgs boson to fermions.

Once the values of the couplings have been chosen, we are able to determine the ratios between the different channels of every decay process. In particular, we must ensure that the SM top decay is the dominant one with respect to the $\mathcal{R}$-parity violating one, and we need to know which one of the two s-bottom decays is the dominant one in order to choose the most convenient parametrisation of branching ratio as described in section 4 Going through the calculations, we obtain for the top quark decay:

$$
\begin{gathered}
\frac{\Gamma\left(t \rightarrow \tilde{\bar{b}}_{1}+\bar{d}\right)}{\Gamma(t \rightarrow W+d)} \approx 0.12 \lambda^{2} \\
\frac{\Gamma\left(\tilde{\bar{b}}_{1} \rightarrow u+d\right)}{\Gamma\left(\tilde{\bar{b}}_{1} \rightarrow \bar{b}+\tilde{\chi}_{1}^{0}\right)} \approx 7.5 \lambda^{2}
\end{gathered}
$$

Where both $\lambda_{313}$ and $\lambda_{323}$ are allowed in the first ratio, and four different couplings $\lambda_{113}, \lambda_{123}, \lambda_{213}$ and $\lambda_{223}$ are allowed in the second ratio. Almost all of them are allowed to be as large as unity. We see that we indeed have, for our values of $\lambda$ couplings, that the B-violating top decay is subdominant, while the ratio between the two possible decays of the s-bottom could be either bigger or smaller than one for different values of $\lambda$, which means that both possibilities described by the equations (12) and (13), or (14) and (15), are actually possible.

We consider now the evolution of these processes in the expanding universe after the EW phase transition. We have already pointed out that the two basic requirements for the viability of this model are the longevity of the top quarks in the early universe, and the out-of-equilibrium feature of their decay. Let us fix, for illustrative purposes, the temperature after the EW 
transition has occurred to $150 \mathrm{GeV}$, then, if we want the top quark to decay at that temperature in a non-equilibrium regime, we must ensure:

$$
\frac{\Gamma(t \rightarrow W+d)}{H_{T_{p . t .}}} \approx \frac{m_{P l}}{2 \cdot 10^{5} \mathrm{GeV}} \leq 1
$$

that translates into:

$$
m_{P l} \leq 2 \cdot 10^{5} \mathrm{GeV}
$$

where $H$ is given by $H^{2}=8 \pi^{3} g_{*}^{1 / 2} T^{2} / 90 m_{P l}$, with $g_{*} \approx 110$ being the number of relativistic degrees of freedom at that time. We have substituted $\Gamma(t \rightarrow W+d)$ for the total the total decay width of t-quark, since this is very accurately true, and we have begun from checking of inequality (26) because this automatically gives to the top and antitop quarks a long-enough life, since in this case the condition $t_{\text {p.t. }} \Gamma(t \rightarrow W+d) \leq 1 / 2$ holds. Equation (27) gives the basic requirement for this model to work, as it has been discussed in section 2 .

We now can compute all the other interesting $\Gamma / H$ ratios, and consequently the baryon asymmetry generated by the processes we have considered so far. We know that we can approximate the kinetic equations for the processes of interest in different ways, which are selected by the value of the ratio $\Gamma / H_{m}$ where $H_{m}=H(T=m), m$ being the mass of the decaying particle, see refs. 4, 22. Given all the numerical values specified above, we obtain:

$$
\begin{aligned}
\frac{\Gamma(t \rightarrow W+d)}{H_{m_{t}}} & \approx \frac{m_{P l}}{3 \cdot 10^{5} \mathrm{GeV}} \\
\frac{\Gamma\left(t \rightarrow \tilde{\bar{b}}_{1}+\bar{d}\right)}{H_{m_{t}}} & \approx \lambda^{2} \frac{m_{P l}}{2.5 \cdot 10^{6} \mathrm{GeV}} \\
\frac{\Gamma\left(\tilde{\bar{b}}_{1} \rightarrow \bar{b}+\tilde{\chi}_{1}^{0}\right)}{H_{m_{\tilde{b}}}} & \approx \frac{m_{P l}}{5 \cdot 10^{6} \mathrm{GeV}} \\
\frac{\Gamma\left(\tilde{\bar{b}}_{1} \rightarrow u+d\right)}{H_{m_{\tilde{b}}}} & \approx \lambda^{2} \frac{m_{P l}}{10^{6} \mathrm{GeV}} \\
\frac{\Gamma\left(\tilde{\chi}_{1}^{0} \rightarrow u+d+d\right)}{H_{m_{\tilde{\chi}}}} & \approx \lambda^{2} \frac{m_{P l}}{10^{12} \mathrm{GeV}}
\end{aligned}
$$

All the above expressions show that if condition (27) is satisfied then automatically it would set these ratios (much) below unity. This in turn 
means that the baryon asymmetry, up to some subsequent dilution processes through entropy production, is simply given by:

$$
\beta \approx 10^{-2} \Delta B
$$

where $\Delta B$ is given by the formulas of section 4 . Whilst every different specific value of the couplings $\lambda$ and the other parameters (mixings and so on) gives different $\Delta B$, we notice that there are several appealing possibilities which could easily deal with the experimentally observed value of $\beta$, without requiring unnatural high values of the various CP-violation parameters involved, $\epsilon \approx 10^{-5}$, even without allowing $\mathrm{CP}$ violation in the SM decay $t \rightarrow W+b$. In this connection we notice that despite the fact that up to date no Electric Dipole Moments have been detected for neutrons or electrons, thus disfavoring maximal $\mathrm{CP}$ violating phases in the MSSM, nevertheless the upper bounds 2] could be satisfied also for large values of the CP violating phases, provided that some cancelation mechanisms are at work, see ref. [23] and references therein.

As a final remark in this section, let us note that the decay of the neutralinos, given the expression (32), would take place at temperatures far below BBN. However, the above mentioned decay width is no longer correct below the scale at which $m_{P l}$ recovers its classical value, which must be before the nucleosynthesis, that is, according to [6], around 10 to $100 \mathrm{MeV}$. Once $m_{P l}$ reaches $10^{19} \mathrm{GeV}$ the decay of the neutralino proceeds almost instantaneously (in comparison with the cosmological expansion rate), thus preventing possible troubles with late-time entropy production which could destroy the successful BBN predictions. Then, since we don't specify how the Planck mass goes up to its standard value or, in other words, which is the time scale for this process, we don't know whether neutralino decays are in equilibrium or not. However this will not change much the results of the scenarios outlined above, since in most of them the asymmetry produced by their decays contributes only a small fraction to the total asymmetry, except for the possibilities given by eq. (10) and (13).

\section{Baryon nonconservation in particle physics}

As we have mentioned above, one of the main goals of this work to present a model of baryogenesis which is capable to explain the observed asymmetry (11) and simultaneously does not contradict very strict lower bound on the proton life-time. As we see below in this section, this model with the chosen parameters values leads to noticeable, but still below the existing limits, neutron-antineutron oscillations and potentially observable nonconservation 
of baryonic charge in heavy quark decays. According to [2] the characteristic time of oscillations for free neutrons is restricted by

$$
\tau_{n \bar{n}}=1 / \delta m_{n \bar{n}}>8.6 \cdot 10^{7} \mathrm{sec},
$$

while for bound neutrons the limit is slightly stronger:

$$
\tau_{n \bar{n}}>1.3 \cdot 10^{8} \mathrm{sec}
$$

The latter is obtained from the observed stability of different nuclei at the level of $10^{32}$ years. If a bound neutron can turn into antineutron inside a nucleus the latter would definitely explode. Non-observation of this process allowed to put the above quoted limit. The arguments go as follows. The admixture of antineutron to neutron in a nuclei is equal to

$$
\sin \theta_{n \bar{n}}=\delta m / \Delta E
$$

where $\delta m$ is the amplitude of $(n-\bar{n})$-transition in vacuum and $\Delta E \approx 100$ $\mathrm{MeV}$ is the energy difference of antineutron and neutron in a nuclei.

The probability of $\bar{n}$ annihilation in a nucleus is equal to

$$
\Gamma_{n \bar{n}} \approx \sigma_{n \bar{n}} V N \sin ^{2} \theta_{n \bar{n}}
$$

where $\sigma_{n \bar{n}}$ is the $n \bar{n}$ annihilation cross section, $V$ is relative velocity of $n$ and $\bar{n}$ and $N \sim m_{\pi}^{3} \sim(100 \mathrm{MeV})^{3}$ is the number density of nucleons inside a nucleus; $\sigma_{n \bar{n}} V \approx 1 / m_{\pi}^{2}$. Assuming, according to the data, that the life-time of nuclei is bounded by $\tau(A \rightarrow A-2)=1 / \Gamma_{n \bar{n}}>10^{32}$ years we find

$$
\tau_{n \bar{n}}=1 / \delta m>(1 / \Delta E) \sqrt{\sigma_{n \bar{n}} V N} \sqrt{10^{32} \text { years }}
$$

Substituting here numerical values indicated above we obtain the result which is incidentally quite close to the limit obtained for free neutrons (35).

In the model under consideration the simplest process which could lead to baryon-antibaryon transformation is (23). On a slightly more fundamental level it is described by a tree diagram of $(u d)$-transition into $\tilde{\bar{b}}_{1}$. Then the latter captures another $d$-quark and turns into $\tilde{\chi}_{1}^{0}$. After that the process goes in charge conjugated order: $\tilde{\chi}_{1}^{0}$ transforms into $\tilde{b}_{1}$ and $\tilde{b}_{1}$ goes into $(\bar{u} \bar{d})$.

The amplitude of this process is

$$
A_{B \bar{B}}=\frac{\lambda_{112}^{2} g^{2} Z_{\tilde{\chi}_{1}^{0}}^{2} Z_{\tilde{b}_{1}}^{2}}{m_{\tilde{b}_{1}}^{4} m_{\tilde{\chi}_{1}^{0}}}(\psi C \psi)^{3},
$$

where $C$ is the charge conjugation matrix and $(\psi C \psi)^{3}$ is the product of 3 spinors of initial state quarks and 3 of the final state antiquarks. The process 
of neutron-antineutron oscillations would be very efficient if $u d d$ quarks (and antiquarks) belonged to the first generation. However, this is not so and the simplest process includes $u$ and $d$ quarks of the first generation and another $d$ quark of the second generation, i.e. the strange quark $s$. Neutron-antineutron oscillations must include transformation with change of strangeness by two units, $\Delta S=2$, and may proceed with additional second order weak interactions, see ref. 25.

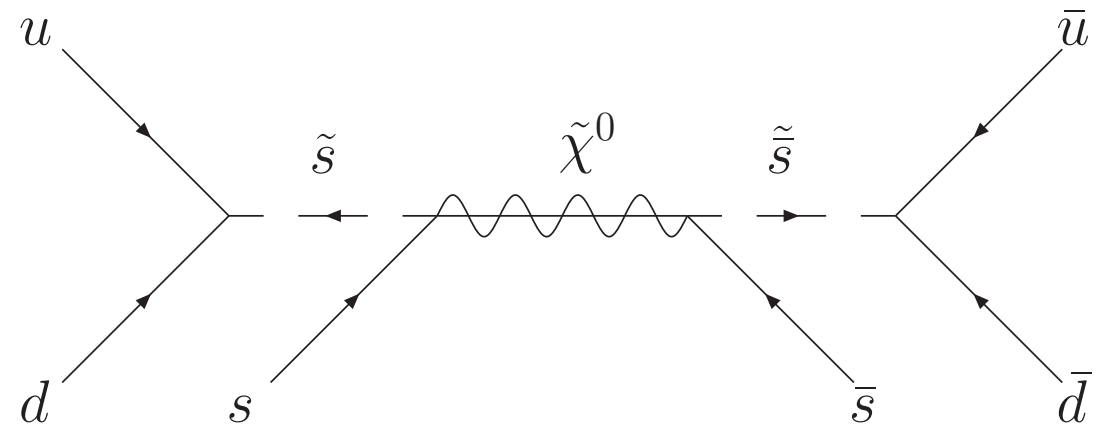

Graph 1. Feynman diagram leading to $n \bar{\Xi}$ transformation or to $\Lambda \bar{\Lambda}$-oscillations. Here every quark is labeled with its proper name.

We can do the same exercise, which is done above for $n \bar{n}$-transformation, considering e.g. the process of neutron transformation into $\bar{\Xi}$-hyperon. A stringent upper bound, $\lambda_{112}<10^{-6}-10^{-7}$, was obtained from nuclei stability with respect to this process in ref. [24] (see also the review [15]). A similar process would create $\Lambda \bar{\Lambda}$-oscillations. Using the same arguments as presented above we find that the life time of $\Lambda \bar{\Lambda}$-oscillations is bounded by approximately $\tau_{\Lambda \bar{\Lambda}}>10^{6}$ sec. A weaker bound in comparison with $n-\bar{n}$ case is related to a larger energy difference of $\bar{\Xi}$ in comparison with $\bar{n}$ and to a smaller annihilation cross-section of $n \overline{\bar{\Xi}}$.

Assuming the specified above mechanism for baryonic number nonconservation we can naively estimate the time of $n \bar{n}$-oscillation using the following chain of processes: neutron transforms into $\Lambda$ by first order weak interaction, then $\Lambda$ goes into $\bar{\Lambda}$ through the process described above and and $\bar{\Lambda}$ "returns" to antineutron again by weak interactions. According to that the time of $n \bar{n}$-oscillations is equal to

$$
\tau_{n \bar{n}} \sim \tau_{\Lambda \bar{\Lambda}} \frac{\left(m_{\Lambda}-m_{n}\right)^{2}}{\mu_{n \Lambda}^{2}}
$$

where $m_{n}$ and $m_{\Lambda}$ are the masses of neutron and $\Lambda$ respectively and $\mu_{n \Lambda}$ is the matrix element or weak transition of neutron to $\Lambda$. The latter can be 
expressed through the amplitude of the decay of $\Lambda \rightarrow p \pi^{-}$, using PCAC and current algebra [26]:

$$
\mu_{n \Lambda} \approx A_{\Lambda \rightarrow p \pi^{-}} / m_{\pi},
$$

where $A_{\Lambda \rightarrow p \pi^{-}}$is the amplitude of the indicated decay of $\Lambda$, which can be easily found from the life-time of $\Lambda$ (we neglected $s-p$ wave problem because it is sufficient for an order of magnitude estimate presented here. Thus we find:

$$
\tau_{n \bar{n}} \sim 10^{12} \tau_{\Lambda \bar{\Lambda}}
$$

This is by far beyond any existing and future experimental sensitivity. The weakness of the $(n \bar{n})$-transformation is related to the necessity of the second order strangeness changing weak transition with $\Delta S=2$.

One can avoid the weak processes with $\Delta S=2$ allowing diagrams with the exchange of superpartner of $W$-boson, wino, $\tilde{W}$, instead of $\tilde{\chi}_{1}$, which permits the vertex $W \rightarrow u \bar{s}$. Such diagrams lead to the amplitude (uuddds) suppressed only by the Cabibbo-like mixing $\sin \tilde{\theta}_{c}$ between wino and strange super-quark plus u-quark. Hence the amplitude of (uudddd)-transition is suppressed only by first order weak interaction. But the effect is still weak. However, even this is not necessary and the weak processes with $\Delta S=1$ can be also avoided leading to the allowed time of $n \bar{n}$-oscillations just above the existing bounds. This makes further improvement of the accuracy in search for this process quite promising.

The mechanism which could lead to noticeable $n \bar{n}$-oscillations is the following. Let us consider the Feynman diagram similar to Graph 1 but with substitution of zino, $\tilde{Z}$, instead of neutralino, $\tilde{\chi}_{1}$. Now the superpartner of s-quark, $\tilde{s}$, can transform into non-strange quark $d$ emitting $\tilde{Z}$. This process was impossible with $Z$-boson due to unitarity of the mass matrix of quarks. Because of that the transformation matrix $Z \bar{q} q$ was always proportional to unity matrix both in the mass and flavour eigenstate basis. This is not necessarily true in the case of non-diagonal transition of quark and its superpartner. Though the mass matrices of quarks and s-quarks are unitary as well, but they can be different for quarks and their superpartners. This allows for the strangeness changing processes in "neutral current" interaction with an exchange of zino 27. Correspondingly the amplitude (uudddd) would be suppressed with respect to (uuddss) only by a mild factor $\sin ^{2} \tilde{\theta}_{c}$, where $\tilde{\theta}_{c}$ is Cabibbo-like mixing angle for s-quark mass matrix. In fact this mixing angle may be even not as small as the Cabibbo angle in usual strangeness charging decays. This opens window for quite strong $n \bar{n}$ mixing.

Let us briefly mention baryonic charger nonconservation in heavy quark decays induced by interaction (667). These processes are of course discussed 
in the literature, see e.g. review [15]. Our only input here is that the constants $\lambda_{i j k}$, connecting heavy $(t, b)$ generation with lighter ones, cannot be as

small as e.g. $\lambda_{112}<10^{-6}$. Otherwise, the baryogenesis in this model would not be efficient enough. Unfortunately we cannot predict how small $\lambda_{i j k}$ are allowed to realize still successful baryogenesis because of uncertainty in the value of the effective Planck mass and mass spectrum of SUSY particles. We could find the range of couplings, masses, and CP-violating phases which are necessary for successful baryogenesis to predict possible range of magnitudes of B-nonconservation effects in particle physics but this is a complicated task which we will postpone.

\section{Conclusion}

We have discussed here a scenario of baryogenesis based on a SUSY model with broken $\mathcal{R}$-parity in such a way that only baryonic charge is noticeably nonconserved, while leptonic charges are either conserved or their nonconservation is very weak.

In the model presented here the baryon asymmetry of the universe can be produced with a sufficiently large magnitude to agree with observations but this demands low scale gravity to ensure the necessary deviations from thermal equilibrium.

On the other hand, proton would not decay despite nonconservation of the baryon number because the decay demands nonconservation of leptonic charge, $L$, which may be arbitrary weak. In the spirit of the mentioned above hierarchy of baryo-nonconserving couplings grossly increasing from light to heavy generations we may assume similar picture for lepton nonconserving couplings. If it is true, lepton nonconservation would be weak in electronic and muonic sector but could be much stronger in tauonic sector. Lepton number nonconserving decays of $\tau$ would be an interesting feature to search for. If leptonic charge is noticeably nonconserved only in $\tau$-sector, proton decay would be suppressed, even with nonconserved leptonic charge.

Still there are several B-nonconserving effects (with conserved $L$ ) which may be observable in direct experiments. Neutron-antineutron oscillations may have the characteristic time just above the existing upper bounds or particles involving heavy quarks could decay into channels with $\Delta B=2$. Unfortunately the predictions are not accurate and theoretical uncertainty may be as large as several (3-4 or more) orders of magnitude due to poor knowledge of the coupling constants, $\lambda$, necessary for baryogenesis.

If $\mathcal{R}$-parity is indeed broken in this way, then the lightest SUSY particle (LSP) should be generally unstable and cannot make the cosmological dark 
matter (DM). We are not completely sure if, in these frameworks, is impossible to make sufficiently long-lived LSP with the life-time bigger than the universe age but have not yet found rigorous arguments either way. If LSP is not DM then other candidates as axion, warm sterile neutrinos, primordial black holes, etc could be DM.

There are several problems yet to study. An interesting one is an investigation of proton decay in a model with heavy Majorana neutrino which is responsible for light neutrino masses. Another thing which could be done is a more accurate study of kinetics of generation of baryon asymmetry with different choices of the (s)particle mass spectrum. This however, is rather an academic problem because we do not know the appropriate parameters (couplings and masses) and accurate calculation with unknown numbers are not particularly interesting, at least at this stage.

\section{Acknowledgments}

We are grateful to Yu. Kamyshkov for discussion and suggestions. A.D. thanks G. Kane for discussion. F.U. is grateful to the Particle Theory Group of the University of Nottingham, for the kind hospitality during the last stage of this work, and thanks O. Seto for useful correspondence. F.U. is supported by INFN under grant n.10793/05.

\section{References}

[1] A. De Rujula, arXiv:astro-ph/9705045.

[2] S. Eidelman et al., Physics Letters, B592 (2004) 1.

[3] A.D. Sakharov, Pis'ma Zh. Eksp. Teor. Fiz. 5 (1967) 32; English translation JETP Lett. 5 (1967) 24.

[4] A. D. Dolgov, Phys. Repts. 222 (1992) 309.

[5] A.G. Cohen, D.B. Kaplan, A.E. Nelson, Ann. Rev. Nucl. Sci. 43 (1993) 27 ;

V.A. Rubakov, M.E. Shaposhnikov, Usp. Fiz. Nauk, 166 (1996) 493;

English translation: Phys. Usp. 39, (1996) 461;

A.D. Dolgov, Surveys in High Energy Phys. 13 (1998) 83;

A. Riotto, M. Trodden, Ann. Rev. Nucl. Part. Sci. 49 (1999) 35;

M. Dine, A. Kusenko, Rev. Mod. Phys. 76 (2004) 1. 
[6] N. Arkani-Hamed, S. Dimopoulos, G. Dvali, Phys. Lett. B429 (1998) 263

I. Antoniadis, N. Arkani-Hamed, S. Dimopoulos and G. Dvali, Phys. Lett. B436 (1998) 257;

For a review see:

A. Pérez-Lorenzana, J. Phys. Conf. Ser. 18 (2005) 224, hep-ph/0503177,

I. Antoniadis, CERN-PH-TH-2005-249, hep-ph/0512182.

[7] D. Cremades, L. E. Ibanez and F. Marchesano, Nucl. Phys. B 643 (2002) 93 arXiv:hep-th/0205074;

C. Kokorelis, Nucl. Phys. B 677 (2004) 115 arXiv:hep-th/0207234.

[8] T. Biswas, A. Notari, hep-ph/0511207.

[9] K. Benakli, S. Davidson, Phys. Rev. D60 (1999) 025004;

A. Masiero, M. Peloso, L. Sorbo, R. Tabbash, Phys. Rev. D62 (2000) 063515 hep-ph/0003312;

R. Allahverdi, K. Enqvist, A. Mazumdar, A. Pérez-Lorenzana, Nucl. Phys. B618 (2001) 277.

[10] A.D. Dolgov, A.D. Linde, Phys. Lett. B116 (1982) 329;

E.W. Kolb, A.D. Linde, A. Riotto, Phys. Rev. Lett. 77 (1996) 4290;

E.W. Kolb,, A. Riotto, I.I. Tkachev, Phys. Lett. B423 (1998) 348;

J. Garcia-Bellido, D.Yu. Grigoriev, A. Kusenko, M.E. Shaposhnikov, Phys. Rev. D60 (1999) 123504.

[11] V. Kuzmin, I. Tkachev, JETP Lett. 68 (1998) 271;

V. Kuzmin, I. Tkachev, Phys. Rev. D59 (1999) 123006;

V. Kuzmin, I. Tkachev, Phys.Repts. 320 (1999) 199;

D.J.H. Chung, E.W. Kolb, A. Riotto, Phys. Rev. D59 (1999) 023501;

D.J.H. Chung, E.W. Kolb, P. Crotty, A. Riotto, Phys. Rev. D64 (2001) 043503.

[12] V. Mukhanov, Physical Foundations of Cosmology (Cambridge University Press, Cambridge, UK, 2005).

[13] A.D. Dolgov, Lectures given at International School of Physics "Enrico Fermi": CP Violation: From Quarks to Leptons, Varenna, Italy, 19-29 Jul 2005, hep-ph/0511213 (to be published in the Proceedings).

[14] K. A. Olive, arXiv:hep-ph/9911307;

S. P. Martin, arXiv:hep-ph/9709356

[15] R. Barbier et al., Phys. Rept. 420 (2005) 1 arXiv:hep-ph/0406039. 
[16] Y. Farzan and J. W. F. Valle, Phys. Rev. Lett. 96 (2006) 011601 arXiv:hep-ph/0509280.

[17] L. E. Ibanez and F. Quevedo, JHEP $9910 \quad$ (1999) 001 arXiv:hep-ph/9908305.

[18] G. F. Giudice, Phys. Rev. D 45 (1992) 3177.

[19] D. Langlois, Prog. Theor. Phys. Suppl. $148 \quad$ (2003) 181 arXiv:hep-th/0209261.

[20] M. Bojowald, Living Rev. Rel. 8 (2005) 11 arXiv:gr-qc/0601085.

[21] A.D. Dolgov, Ya.B. Zeldovich, Uspekhi Fiz. Nauk, 130 (1980) 559 (in Russian);

A.D. Dolgov, Ya.B. Zeldovich, Rev. Mod. Phys. 53 (1981) 1.

[22] E. W. Kolb and M. S. Turner, The Early Universe (Addison-Wesley, Redwood City, USA, 1990).

[23] S. Y. Ayazi and Y. Farzan, arXiv:hep-ph/0605272.

[24] R. Barbieri, A. Masiero, Nucl. Phys. B267 (1986) 679.

[25] D. Chang and W. Y. Keung, Phys. Lett. B 389 (1996) 294 arXiv:hep-ph/9608313;

J. L. Goity and M. Sher, Phys. Lett. B 346 (1995) 69 [Erratum-ibid. B 385 (1996) 500] arXiv:hep-ph/9412208.

[26] A.I. Vainshtein, V.I. Zakharov, Usp. Fiz. Nauk 100 (1970) 225; English translation: Sov. Phys. Usp. 13 (1970) 73.

[27] P. H. Chankowski, O. Lebedev and S. Pokorski, Nucl. Phys. B 717 (2005) 190 arXiv:hep-ph/0502076;

D. J. H. Chung, L. L. Everett, G. L. Kane, S. F. King, J. D. Lykken and L. T. Wang, Phys. Rept. 407 (2005) 1 arXiv:hep-ph/0312378. 